\title{
Rational approaches for optimizing chemical functionality of plasma polymers: a case study with ethyl trimethylacetate
}

\begin{tabular}{|r|l|}
\hline Journal: & Plasma Processes and Polymers \\
\hline Manuscript ID & ppap.202000195.R1 \\
\hline Wiley - Manuscript type: & Full Paper \\
\hline Author: & n/a \\
\hline Complete List of Authors: & $\begin{array}{l}\text { saboohi, solmaz; University of South Australia, Future Industries } \\
\text { Institute; } \\
\text { Coad, Bryan; University of Adelaide School of Agriculture Food and Wine } \\
\text { - Waite Campus } \\
\text { Short, Robert; Lancaster University Faculty of Science and Technology } \\
\text { Michelmore, Andrew; University of South Australia, Mawson Institute } \\
\text { Griesser, Hans; University of South Australia, Future Industries Institute }\end{array}$ \\
\hline Keywords: & \multicolumn{2}{|c}{} \\
\hline &
\end{tabular}

\section{SCHOLARONE \\ Manuscripts}




\title{
Rational approaches for optimizing chemical functionality of plasma polymers: a case study with ethyl trimethylacetate
}

\author{
Solmaz Saboohi ${ }^{1}$, Bryan R. Coad ${ }^{1,2}$, Robert D. Short ${ }^{3}$, Andrew Michelmore ${ }^{1,4}$, Hans J.
} Griesser $^{1}$

1. Future Industries Institute, University of South Australia, Mawson Lakes, South Australia 5095, Australia

2. School of Agriculture Food \& Wine, University of Adelaide, South Australia 5005, Australia.

3. Materials Science Institute and Department of Chemistry, University of Lancaster, City of Lancaster, $U K$

4. School of Engineering, University of South Australia, Mawson Lakes, South Australia 5095, Australia

\begin{abstract}
Improved retention of desirable chemical structures during plasma polymerization requires rational tailoring of plasma phase conditions. Using ethyltrimethylacetate, we studied effects of pressure and power on the contribution of intact molecular ions to deposition and retention of ester groups. The abundance of protonated molecular ions in plasmas varies with pressure and power, but the functionality of plasma polymers, assessed by XPS and ToF-SIMS, did not correlate. Together with high ion flux, the ion energy distribution was found to be a key parameter and needs to be tailored to enable ions to soft-land on the surface after traversing the sheath. The compromise between abundance of ions and their energy distribution is optimal near the transition between the $\alpha$ and $\gamma$ plasma phases.
\end{abstract}

Keywords: Plasma regimes, surface functionality, plasma transition point, plasma polymerization, mass spectroscopy, ToF-SIMS, XPS, ETMA, ethyl trimethylacetate 


\section{INTRODUCTION}

Plasma polymerization has found use in a range of industrial applications for reason of its processing advantages in the deposition of thin film coatings. A major drawback, however, is that plasma polymers are not well-defined molecularly with chemical structures akin to conventional polymers that can be produced with high chemical specificity and order. Plasma polymers typically possess a diversity of chemical groups/structures. To mitigate this issue, researchers have used empirical approaches to increase the density of desired chemical groups. The most common approaches involve deposition under very low power conditions [1-5], which then entails a considerable reduction in the deposition rate, and pulsing [1, 6-8], which again leads to markedly reduced deposition rates and the deposits may be soluble [9-11].

While the empirically derived "recipes" of low power and/or pulsing have led to increases in the functionality of plasma polymer films, there is still little understanding of how to optimize plasma conditions in a rational manner to produce high densities of functional groups in and on the surface of plasma polymers. In particular, instead of empirically scanning plasma parameters across a range of values, it would be highly desirable to utilize a strategy that possesses predictive ability and thus reduces the number of experiments necessary to arrive at maximally functionalized plasma polymers.

It appears reasonable to start with the postulate that the diversity of chemical structures in plasma polymers derives from the non-thermodynamic cleavage of various covalent bonds in the "monomer" molecule producing radicals that then can fragment, rearrange, and combine in various ways. Indeed, from early on, polymerizing plasmas were described as chemical "soups" with a rich diversity of radicals and neutrals in the plasma gas phase [12]. However, while most often plasma polymerization has been discussed in terms of radical reactions and combinations, it is also well-established by now that ions can also play important roles in the plasma gas phase and in the build-up of coatings [13-15]. The acceleration of ions across the plasma sheath, versus the diffusion-controlled transport of radical species, means that ions can play a role in plasma polymer build-up that substantially exceeds their relative abundance in the bulk. Moreover, ions may be much less susceptible to rapid reactions in the plasma phase. Thus, if they can be induced to play a major role in the deposition process, the diversity of chemical structures in plasma polymers might be amenable to rational reduction and the desired functionality might be retained and incorporated with maximal density.

Hence, a promising strategy would seem to be to control plasmas in ways that reduce the random nature of radical processes and complementarily enhance the role of ions. In recent work [16-18] we have shown that pressure plays a key role; with several monomers, mass 
spectrometry analysis showed that near the transition between $\alpha$ and $\gamma$ plasma gas phases a high relative density of positive ions resulted, particularly of the protonated molecular ions. Coatings deposited under such conditions exhibited high extents of desired functionalities.

For the sake of clarity, we stress that in such cases, plasma polymer deposition does not arise only from ionic contributions; the various radical species produced in the plasma play an essential role in assembling the growing films whilst ions primarily contribute the desired chemical functionality. Monomer activation in the plasma phase yields radicals and heavy ions that together determine the film composition. Hence the task is to optimize the contribution of ions that have retained the desired chemical group through plasma activation, whilst ensuring that the totality of components produced in the plasma generate an efficient film building process.

However, if ions arrive at the surface of the substrate or the growing film with excessive energy, they will fragment and thereby again lead to multifunctional films. How can a plasma be steered towards producing a high density of desirable ions and also so as to enable transport of those ions across the plasma sheath in a way that minimizes fragmentation? And does this necessarily involve the use of low power or pulsing? Here we report an investigation of the generation of molecular ions and their transport to the surface, using the monomer ethyl trimethylacetate (ETMA) as an illustrative example. We show that the ion energy distribution and the ion flux play key roles, and we discuss ways to optimize them and thereby implement a rational approach to maximising functionality.

\section{EXPERIMENTAL SECTION}

\subsection{Plasma Polymerization.}

Plasma polymer (pp) coatings from Ethyl trimethylacetate (ETMA, 99\%, Sigma -Aldrich) were deposited using a stainless steel vacuum chamber. The plasma reactor used has been described and characterized elsewhere.[16] The chamber was pumped down by a two-stage rotary pump to reach a base pressure below $2 \times 10^{-3}$ mbar. Silicon wafers were used as substrates after solvent-cleaning with ethanol and acetone followed by drying under a stream of dry Nitrogen. ETMA vapour was introduced into the chamber via a ball valve after degassing using liquid nitrogen. Then plasma was ignited using an RF power generator (13.56 MHz, RFG050 Coaxial Power Systems, UK) with a matching network (AMN150, Coaxial Power Systems, UK). Coatings were deposited at RF input power of $50 \mathrm{~W}$ and $10 \mathrm{~W}$, under different pressure values across the collision-less and collisional plasma regimes. 


\subsection{Plasma Mass Spectrometry (Plasma MS)}

A quadrupole mass spectrometer (Hiden EQP1000 energy resolving mass spectrometer) was mounted along the reactor midline axis. The system was differentially pumped during operation using a turbomolecular pump. The internal pressure remained below $5 \times 10^{-7} \mathrm{mbar}$. A grounded $100 \mu \mathrm{m}$ orifice was used during sampling. The mass spectrometer was operated in residual gas analysis (RGA) and positive ion modes to acquire spectra of both plasma-phase neutral species and ions, respectively. The ion optics were tuned to give a maximum signal at the molecular weight of $131 \mathrm{~m} / \mathrm{z}$ for ETMA. The ion energy distribution of the molecular ion at different RF powers was measured. In positive ion mode, the ion optics were first tuned to the peak ion energy at each power for the protonated precursor by acquiring the ion energy distribution. The positive ion mass spectra were then collected at this peak ion energy in the range 0-300 $\mathrm{m} / \mathrm{z}$.

\subsection{X-ray Photoelectron Spectroscopy (XPS)}

XPS was used to determine the chemical composition of the surface layers of the ETMA plasma polymer films. Samples were analyzed using a Kratos Axis Ultra DLD spectrometer equipped with a monochromatic Al Ka source. Samples were analyzed at a photoelectron emission angle normal to the sample surface. Survey spectra were acquired at $120 \mathrm{eV}$ pass energy and high-resolution $\mathrm{C} 1 \mathrm{~s}$ spectra were recorded at $20 \mathrm{eV}$ pass energy. Spectra with high resolution were recorded for $\mathrm{C}$ and $\mathrm{O}$ peaks. Quantifications of atomic percentages and curve fitting analysis were performed using Casa XPS software (ver. 2.3.16 Pre rel. 1.4, Casa Software Ltd.). Spectra were charge-corrected by offsetting the binding energy relative to the $\mathrm{C}-\mathrm{C}$ component of the $\mathrm{C} 1 \mathrm{~s}$ spectrum, which was set to $285.0 \mathrm{eV}$.

\subsection{Static Time-of-Flight Secondary Ion Mass Spectrometry (ToF-SIMS)}

ToF-SIMS analyses were performed using a Physical Electronics Inc. PHI TRIFT V nanoToF instrument equipped with a pulsed liquid metal $79 \mathrm{Au}^{+}$primary ion gun (LMIG) operating at an energy of $30 \mathrm{kV}$. Surface analyses were performed using "bunched”' Au1 beam settings to optimize mass resolution. Spectra were collected in positive SIMS mode using a $100 \mathrm{x} 100$ $\mu \mathrm{m}^{2}$ raster area with acquisition times of $1 \mathrm{~min}$. Six independent spectra were recorded per sample from separate spots, at an ion dose of $1 \times 10^{11}$ ions $/ \mathrm{cm}^{2}$. A mass resolution $\left({ }^{M} / \Delta M\right)$ of 6500-7000 was typically achieved at nominal m/z $27\left(\mathrm{C}_{2} \mathrm{H}_{3}{ }^{+}\right)$. Spectra were calibrated using the $\mathrm{CH}_{3}{ }^{+}, \mathrm{C}_{2} \mathrm{H}_{5}{ }^{+}$, and $\mathrm{C}_{3} \mathrm{H}_{7}{ }^{+}$peaks. The peak intensities in ToF-SIMS spectra are dependent on the experimental conditions; therefore, each peak was normalized to the total counts of the corresponding spectrum. Experiments were performed under a vacuum of 5 x $10^{-6} \mathrm{~Pa}$.

\subsection{Ion Flux}


The ion flux to the RF electrode was measured using an Impedans OctIV ion flux probe (Impedans, Dublin) placed in series between the matching network and the RF electrode. The ion flux for a collision-less sheath is given by the Bohm flux,

$J_{i}=\exp \left(-\frac{1}{2}\right) n_{i} \sqrt{\frac{k T_{e}}{m_{i}}}$

Where $n_{i}$ is the plasma density, $k$ is Boltzmann's constant, $T_{e}$ is the electron temperature and $\mathrm{m}_{\mathrm{i}}$ is the ion mass. For a homogeneous plasma, the ion flux is constant to all surfaces in contact with the plasma, thus the ion flux to the RF electrode equals the ion flux to the counter electrode (wall area). Therefore, the ion flux to the substrate was taken to accord with the measured ion flux to the RF electrode [19-22].

2.6 Quartz Crystal Microbalance (QCM)

A Quartz Crystal Microbalances (QCM) manufactured by Sycon Instruments, was used to measure the deposition rate during plasma polymer film deposition. It consisted of a $7 \mathrm{~mm}$ diameter $6 \mathrm{MHz}$ gold crystal in the centre of the bottom electrode. The silicon wafer substrates were placed as close as practicable to the QCM sensor during plasma polymerization experiments [20].

\section{RESULTS AND DISCUSSION}

Our objective in the plasma polymerization of ETMA was the retention of the ester group. Thus, in the plasma gas phase there should be minimal cleavage of the $\mathrm{C}-\mathrm{O}$ and $\mathrm{C}=\mathrm{O}$ bonds and plasma polymerization proceeding by replacing, preferably, some of the $\mathrm{C}-\mathrm{H}$ bonds with new C-C bonds. While some of this is likely to occur in the plasma gas phase, our focus was on investigating how best to form the protonated molecular ion $\left([\mathrm{M}+\mathrm{H}]^{+}\right)$and to transport it through the sheath, so that it would play a major role in the formation of the growing plasma polymer film and thereby confer a high extent of the desired functionality. Enhancing the role of molecular ions would seem an approach that lends itself better to understanding and control than the radical pathway of plasma polymerization. Yet, we stress that ETMA activation in the plasma phase yields radicals and heavy ions that together determine the film composition, but it is the latter that are capable of maximizing functionality.

\subsection{Effect of pressure}

We have shown previously by mass spectrometry that in plasmas of other ester monomers, a high density of protonated molecular ions (and other positively charged ions) can be obtained when the pressure is selected to be near the transition between the $\alpha$ and $\gamma$ plasma 
phases $[17,18]$. Accordingly, we first investigated whether this would also apply with ETMA. Figure 1 reproduces plasma phase mass spectra recorded during plasma polymerization at three different pressures and an RF power input of $50 \mathrm{~W}$. The ETMA molecule has a molecular weight of $130.18 \mathrm{Da}$. Thus, the signal at $\mathrm{m} / \mathrm{z} 131$ in the mass spectra shows that the protonated molecular ion is produced with high abundance. It should be noted that there are various pathways to produce the protonated molecular ion [23]; for example they may be produced in the plasma phase via attachment of $\mathrm{H}^{+}$, or due to charge transfer after ion-molecule collisions within the sheath. Regardless of how the ions are produced, though, the mass spectrometer measures the ionic species which reach surfaces in contact with the plasma, and thus contribute to film deposition. The efficient generation of $(\mathrm{M}+\mathrm{H})^{+}$is probably due to two factors: first, the high abundance and promiscuity of $\mathrm{H}^{+}$in plasmas [24] and secondly, resonance stabilization of the charge that arises upon addition of a proton to ester and carboxylic acid groups.

Positive ions assignable to fragments from ETMA are also observed, with relative intensities that vary with the pressure of the plasma phase. The structures of some of those ions are shown in Figure 1.
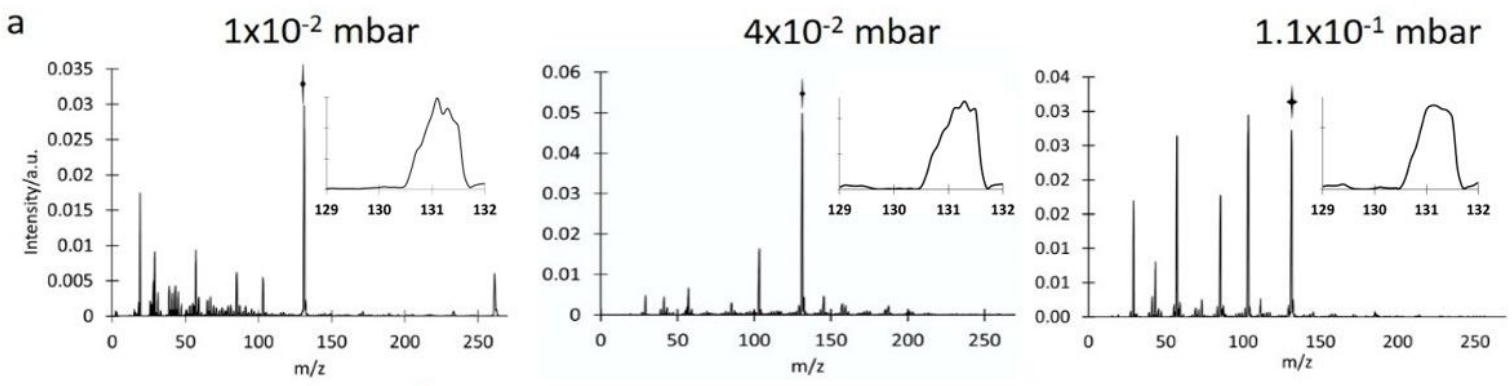

b

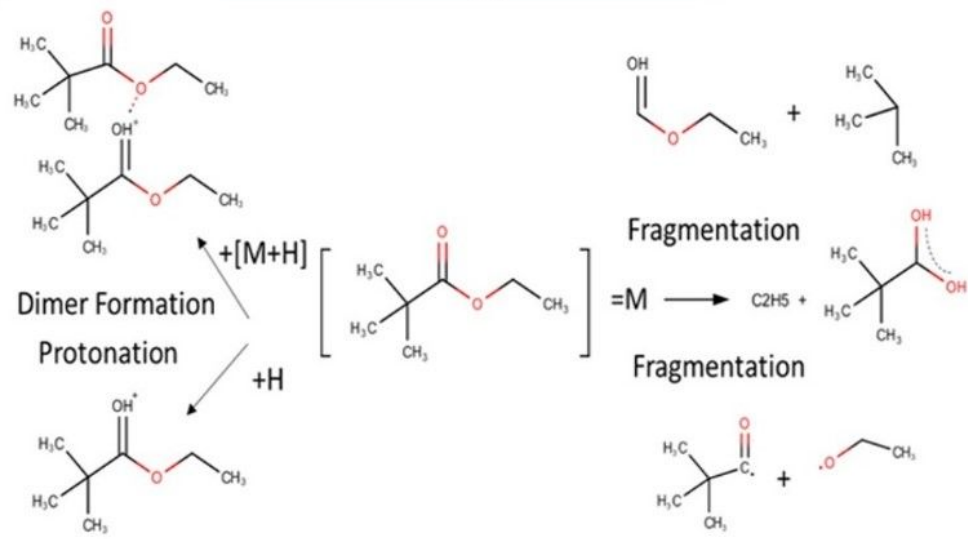

Figure 1. a) Positive ion mass spectra recorded with ETMA plasmas at pressures (from top) of $1 \times 10^{-2} \mathrm{mbar}, 4 \times 10^{-2} \mathrm{mbar}$ and $1.1 \times 10^{-1} \mathrm{mbar}$, and a plasma input power of $50 \mathrm{~W}$. The protonated molecular ion $[\mathrm{M}+\mathrm{H}]^{+}$signal is marked by + . Insets show high resolution signals of the 130 $\left(\mathrm{M}^{+}\right)$and $131\left(\mathrm{M}+\mathrm{H}^{+}\right) \mathrm{m} / \mathrm{z}$ species. b) Major pathways occurring in ETMA plasmas to account for the observed ion signals. For the fragments, ion formation is omitted for clarity. 
Does generation of these ions to appreciable amounts in the plasma phase translate to a high extent of functionality in the plasma polymer films produced at these pressures? If not, why not? Accordingly, we assessed the chemical composition of the coatings by XPS and ToFSIMS. High-resolution XPS C1s spectra are shown in Figure 2A. It is evident that the plasma polymers are of predominantly hydrocarbon composition. Some intensity is manifest for C-O and $\mathrm{O}-\mathrm{C}=\mathrm{O}$ components, but clearly the extent of retention of the ester functionality is relatively low. The highest intensity of the $\mathrm{O}-\mathrm{C}=\mathrm{O}$ component was observed for the plasma polymer deposited at $4 \times 10^{-2}$ mbar, which is near the transition between the $\alpha$ and $\gamma$ phases.
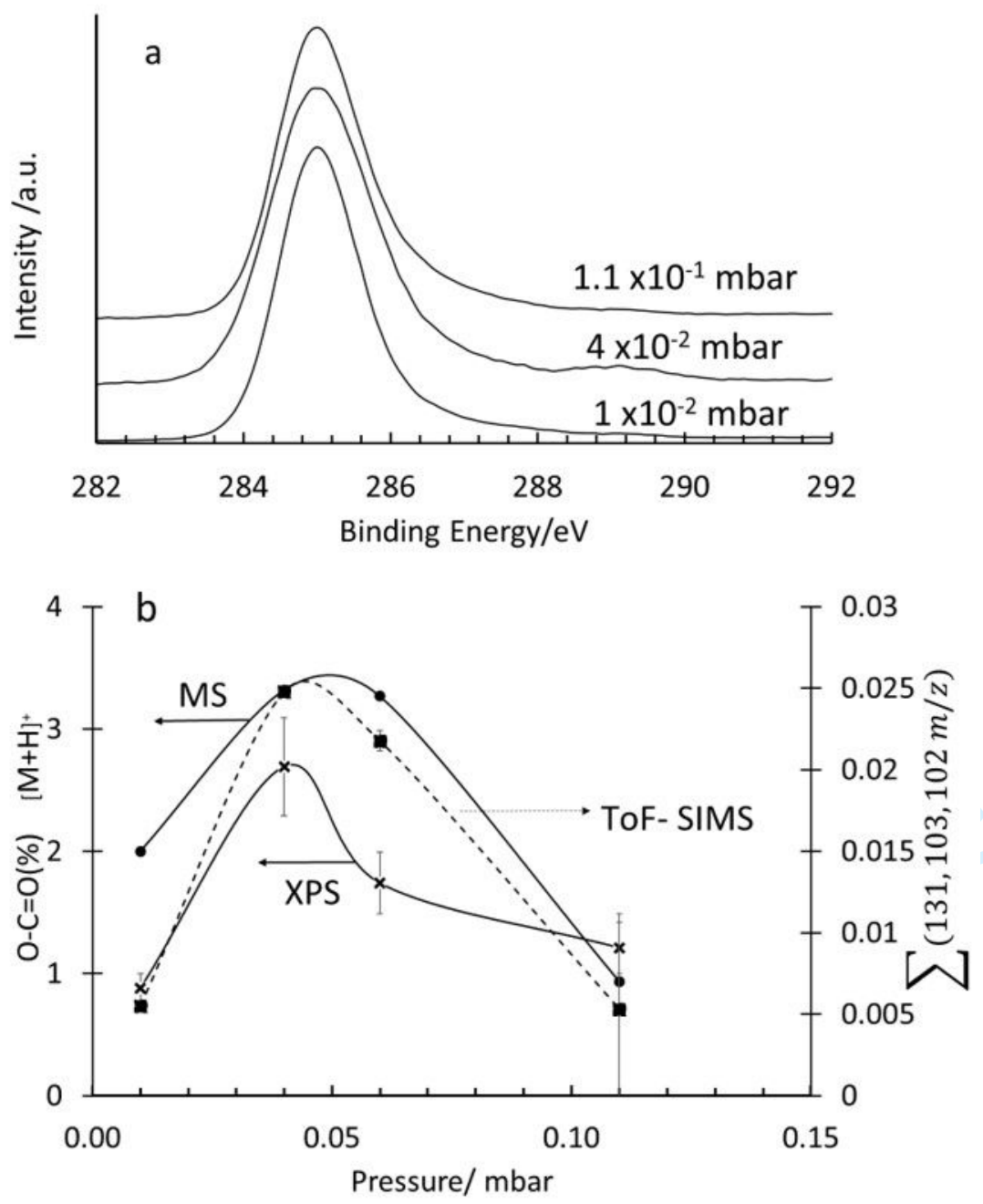

Figure 2. a) XPS C1s spectra recorded with ETMA plasma polymers deposited at pressures (from top) of $1 \times 10^{-2} \mathrm{mbar}, 4 \times 10^{-2} \mathrm{mbar}$ and $1.1 \times 10^{-1} \mathrm{mbar}$, and a plasma input power of $50 \mathrm{~W}$. b) Normalized intensities of the $[\mathrm{M}+\mathrm{H}]^{+}$ion in plasma-phase $\mathrm{MS}$, the $\mathrm{O}-\mathrm{C}=\mathrm{O}$ component in C1s spectra, and the combined signals $\mathrm{m} / \mathrm{z}$ 131, 103 and 102 in ToF-SIMS spectra, as a function of the pressure during plasma polymerization. 
Positive ion ToF-SIMS spectra (not shown) were also recorded for the plasma polymers deposited at the three different pressure values. Signals of $\mathrm{m} / \mathrm{z} 131,103$ and 102 arise from ions containing the ester motif and thus allow comparison of extents of incorporation into the plasma polymers. The combined normalized intensities (normalized to the sum of the intensities of all ion signals) are shown in Figure 2B, which also contains the normalized intensities of the protonated molecular ion in plasma MS and of the $\mathrm{O}-\mathrm{C}=\mathrm{O}$ component in XPS C1s spectra.

Figure 2B reveals two interesting conclusions. First, the three data sets correlate quite well, indicating that the higher the abundance of the $[\mathrm{M}+\mathrm{H}]^{+}$ion is in the plasma phase, the higher the extent of incorporation is of the desired ester functionality in the plasma polymer coatings. We recognize that the $\mathrm{O}-\mathrm{C}=\mathrm{O}$ component in XPS $\mathrm{C} 1 \mathrm{~s}$ could also contain carboxyl groups, but the good correlation with the other variables suggests that it can be assumed that the signal arises mainly from ester C. Secondly, all three measurables show a maximum in the pressure region where the plasma transitions between collision-less and collisional regimes.

Therefore, we can conclude that the highest extent of functionality in ETMA plasma polymers is achieved when selecting the pressure to be near the plasma phase transition. Yet, the extent of retention of functionality in all these plasma polymers is disappointingly low, as shown best by the XPS C1s spectra. Why is this so, and can we do better?

As discussed in the introduction, the production of ions may be a useful first criterion, but evidently is not sufficient per se. Ions that traverse the plasma sheath and arrive at the surface (first of the substrate and then the growing film) with high kinetic energy are likely to fragment upon impact. Ions that arrive with a sufficiently low energy can "soft land" and thus add to the growing film intact, conferring the desired molecular structure. When a molecule impacts on a surface, its translational energy largely governs the branching ratio between the various reaction pathways such as molecular physisorption (dominant at $0.1-1 \mathrm{eV}$ ), soft landing, and finally sputtering processes at high kinetic energies [25]. It has been assumed that polyatomic ions with energy below 15 to $25 \mathrm{eV}$ may be amenable to soft-landing [26, 27]. We will use below a value of $15 \mathrm{eV}$ as an approximate value for the soft-landing threshold energy.

It seems reasonable to assume that the soft-landing threshold energy should depend on the size and molecular structure of the impinging ions, with larger molecules capable of distributing kinetic energy across a larger number of bonds. This will need to be verified experimentally in future research.

Measurement of the ion energy distribution (IED) may be a revealing way of assessing the effectiveness of translating intact ions from the plasma phase into a growing plasma 
polymer. Figure 3 shows IEDs recorded at three pressures for the $[\mathrm{M}+\mathrm{H}]^{+}$ion. Clearly, at the lowest pressure, from a collision-less plasma, ions arrive at the surface with energies well in excess of the threshold for soft landing, suggesting that there will be a high extent of fragmentation upon impact. This IED suggests a low extent of collisions. At intermediate pressure, near the plasma transition, the ion energies are much reduced and broadened by collisions, but the IED extends up to $30 \mathrm{eV}$ and hence some of the arriving ions still possess an energy that may lead to fragmentation. At the highest pressure, the average energy is reduced slightly further still, but again the IED still extends to $30 \mathrm{eV}$.

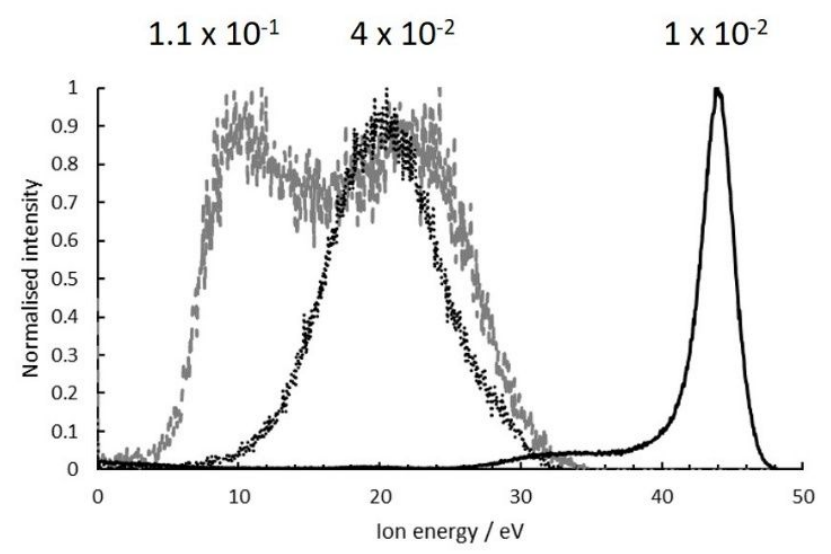

Figure 3. Ion energy distributions of $[\mathrm{M}+\mathrm{H}]^{+}$ions from ETMA plasmas at (from top) $1 \times 10^{-2}$ mbar, $4 \times 10^{-2}$ mbar and $1.1 \times 10^{-1}$ mbar, and a plasma input power of $50 \mathrm{~W}$.

Figure 3 could be taken to suggest that use of the highest pressure setting should give the highest extent of retention of the desired ester functionality, but Figure 2 shows that this is not the case. At the highest pressure there is a higher probability for ions to undergo collisions while traversing the plasma sheath, with such collisions reducing the flux of intact ions arriving at the surface. This is evident in the $y$ axis of the three distributions.

Hence, we can now summarize the pressure dependence of the role of ions as follows. In a collision-less plasma there is efficient production of the desired protonated molecular ion, but these ions arrive at the surface with high energy and thus are likely to fragment upon impact, leading to relatively inefficient incorporation of larger molecular structures such as the ester group in ETMA. With higher pressure plasmas there still are high relative abundances of the protonated molecular ion, but the flux to the surface is reduced by collisions, increasing with increasing pressure. However, their energy distribution is much lower on average, increasing the probability of soft landing. An optimal compromise between these counteracting trends 
appears to arise when selecting a pressure near the transition between the collision-less and collisional plasma regimes.

\subsection{Effect of RF power}

Even under our best conditions, however, ester groups comprise less than $3 \%$ of the total $\mathrm{C}$ in the plasma polymer (Figure 2). Many studies have found that use of low power in plasma polymerization leads to better retention of functionality. Thus, we next report data analogous to those above for plasmas struck with $10 \mathrm{~W}$ power input, using the same analytical methods to identify the key reasons for compositional changes in the plasma polymers. We note that with $50 \mathrm{~W}$ at all pressures, the ion energy distribution is such that many ions possess more energy than we would ideally like. Can this be improved by reducing the RF power input, or are we just reducing the production, or altering the nature, of active species in the plasma?

Plasma mass spectra recorded at various pressures, reproduced in Figure 4, again show that the relative abundance of the various ions varies with pressure. But the spectra are not hugely different from those recorded with $50 \mathrm{~W}$ power input. The signal from the protonated molecular ion again is prominent at all pressures except for the lowest pressure, where substantial fragmentation is evident. The most distinctive feature is the appearance of an intense dimer ion signal in Figure 4B. Dimer formation was observed previously for another plasma [28]. Notwithstanding such differences, for the present purposes we conclude that reducing power does not lead to marked changes in the nature of the ionic species in ETMA plasmas, a conclusion which was not entirely expected in the light of many reports on changing compositions of plasma polymers with applied rf power.
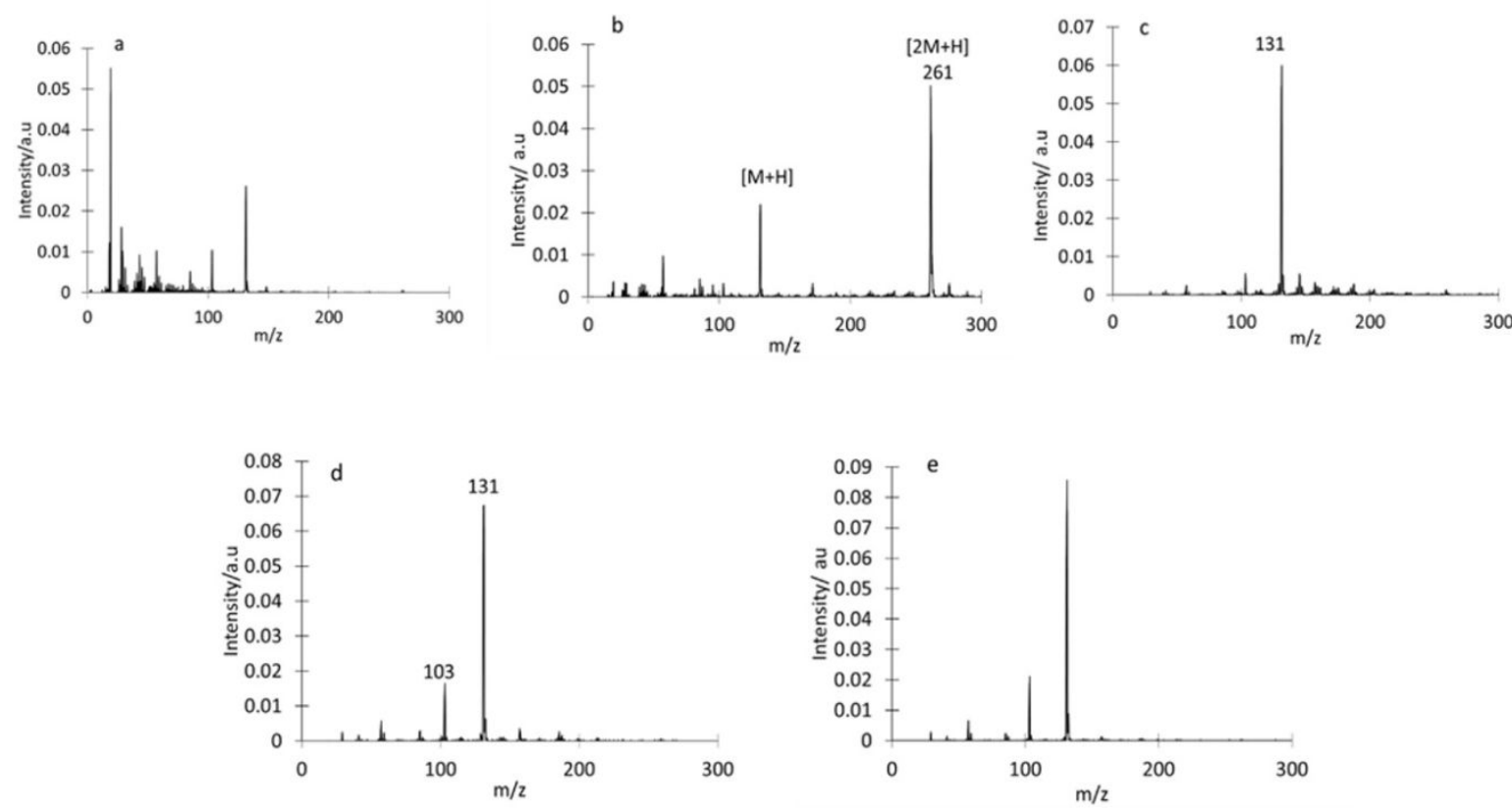
Figure 4. ETMA plasma mass spectra at pressures of a) $7 \times 10^{-3}$ mbar, b) $1.5 \times 10^{-2}$ mbar, c) $4 x$ $10^{-2}$ mbar and d) $8 \times 10^{-2}$ mbar e) $1.1 \times 10^{-1}$ mbar and RF power $10 \mathrm{~W}$.

The ion energy distributions recorded for the protonated molecular ion (Figure 5), on the other hand, differed substantially from those recorded at $50 \mathrm{~W}$. Again, not surprisingly, the highest average ion energy was recorded at the lowest pressure, but its average at $\sim 21 \mathrm{eV}$ was considerably lower than for $50 \mathrm{~W}$ power. Analogously, the other IEDs also are centred at substantially lower values. Operating the plasma near or above the transition pressure between $\alpha$ and $\gamma$ phases yields IEDs that suggest that most ions should soft-land. Again, as for $50 \mathrm{~W}$, the lowest IED was obtained at the highest pressure used, but the additional collisions also reduced the ion flux.

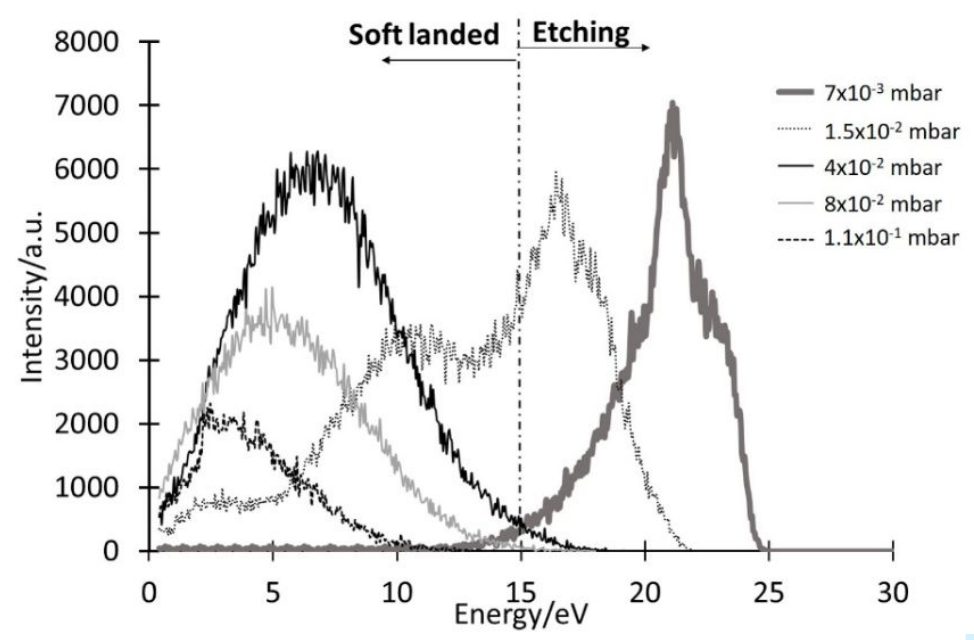

Figure 5. Ion energy distribution of $[\mathrm{M}+\mathrm{H}]^{+}, \mathrm{m} / \mathrm{z} 131$, as a function of pressure, at power $=$ $10 \mathrm{~W}$.

If our hypothesis is correct that soft-landed ions are important for incorporating the desired chemical group into plasma polymers, we now should expect that under those plasma conditions that lead to low ion energies, we should obtain a higher extent of incorporation of ester groups into the plasma polymers. Figure 6 reproduces XPS C1s spectra recorded with plasma polymers produced with $10 \mathrm{~W}$ power and at various pressures. It is evident that again the plasma polymer deposited at the lowest pressure has little ester functionality. At higher pressures, on the other hand, a significant $-\mathrm{O}-\mathrm{C}=\mathrm{O}$ component (at $289 \mathrm{eV}$ ) is clearly visible. Figure 6B shows, for clarity, the component fitting for the plasma polymer deposited at intermediate pressure; this fitting also shows a significant $\mathrm{C}-\mathrm{O}$ component, consistent with 
intact ester groups. Again assuming that the amount of carboxylate groups is low and thus the $-\mathrm{O}-\mathrm{C}=\mathrm{O}$ component in XPS $\mathrm{C} 1 \mathrm{~s}$ largely arises from ester groups, we can conclude that there is indeed significant incorporation of intact ester groups. The fitted intensity of the $-\mathrm{O}-\mathrm{C}=\mathrm{O}$ component is $6.5 \%$ for the plasma polymer deposited at $4 \times 10^{-2}$ mbar, which is a marked increase compared with the intensity of $2.4 \%$ with $50 \mathrm{~W}$ power input (Fig. 2).

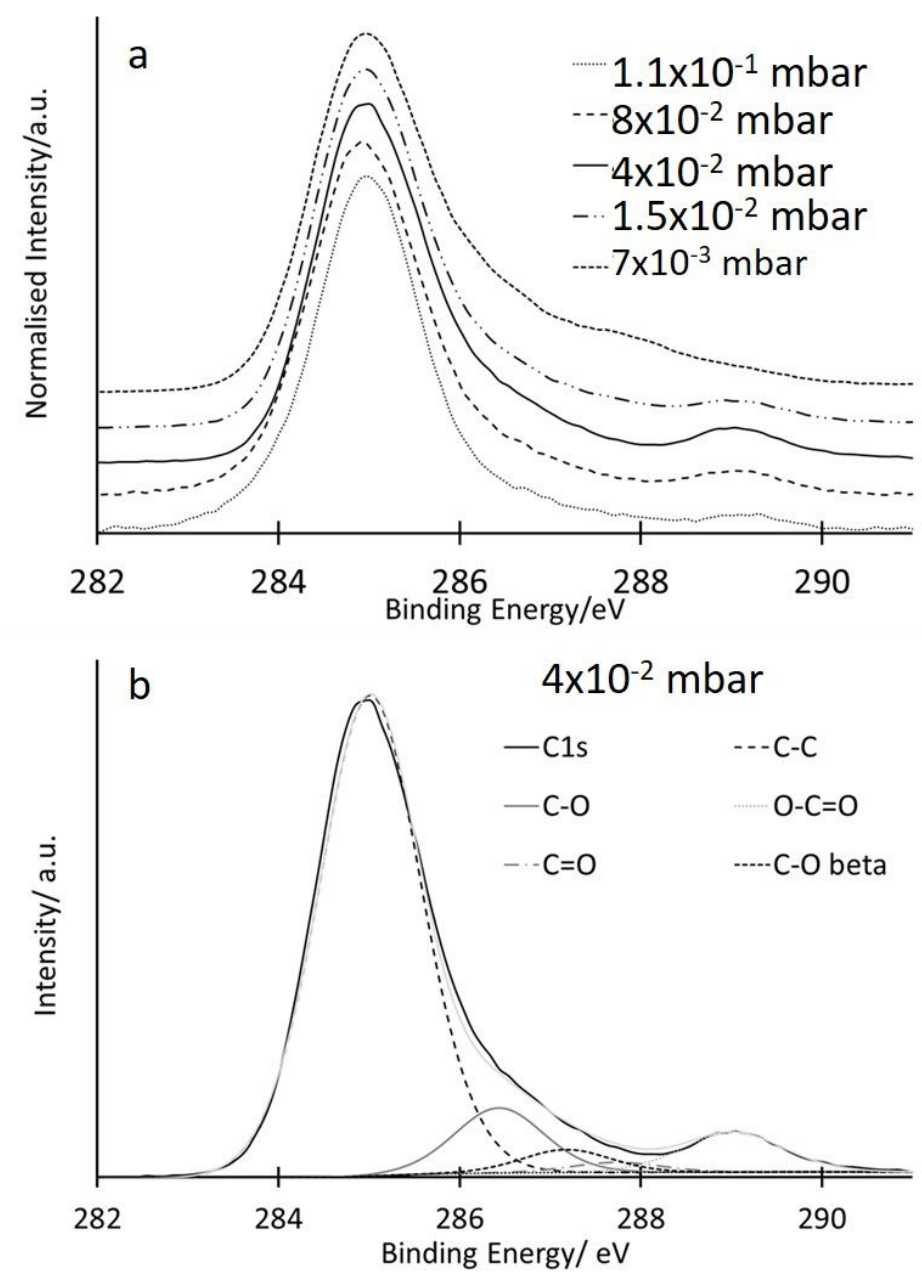

Figure 6. A) XPS C1s spectra for ETMA plasma polymers deposited under various pressures and with $10 \mathrm{~W}$ RF power; B) Component fitting of the C1s spectrum from the plasma polymer deposited at a pressure of $4 \times 10^{-2} \mathrm{mbar}$.

ToF-SIMS enables independent assessment of this point, in that peaks at $\mathrm{m} / \mathrm{z} 131$ (which was the dominant signal for plasma polymers deposited at intermediate pressure values), 103 and 102 are indicative of structures containing the entire monomer molecule and the $-\mathrm{O}-\mathrm{C}=\mathrm{O}$ functionality, respectively. In Figure 7 the sum of the normalized intensities of these peaks is displayed as a function of the pressure. Also plotted are the intensities of the protonated molecular ion in plasma-phase $\mathrm{MS}$ and of the $\mathrm{C} 1 \mathrm{~s}-\mathrm{O}-\mathrm{C}=\mathrm{O}$ component. It is striking 
that the same correlations and the same shape are observed as in Figure $2 \mathrm{~b}$ when $50 \mathrm{~W}$ power input was used; again the intermediate pressure of $4 \times 10^{-2}$ mbar provided optimal conditions.

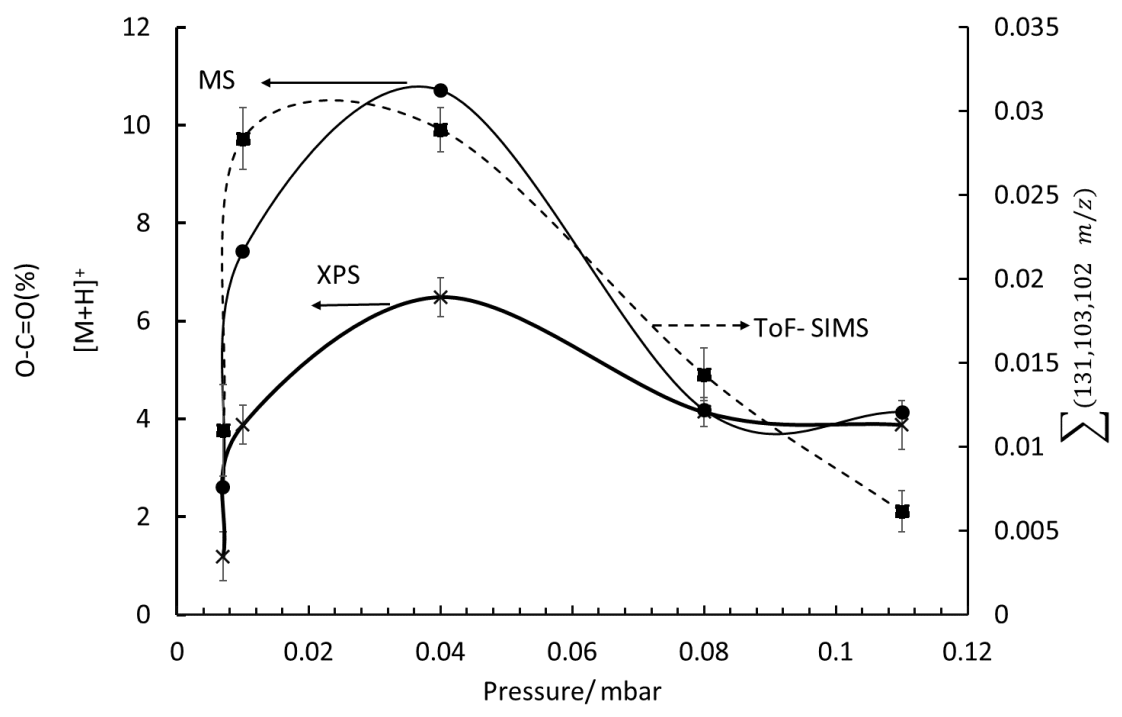

Figure 7. Normalized intensities of the $[\mathrm{M}+\mathrm{H}]^{+}$ion in plasma-phase $\mathrm{MS}$, the $\mathrm{O}-\mathrm{C}=\mathrm{O}$ component in C1s spectra, and the combined signals m/z 131, 103 and 102 in ToF-SIMS spectra, as a function of the pressure during plasma polymerization with a power of $10 \mathrm{~W}$.

Thus, the applied RF power has a marked effect on the extent of incorporation of functionality into our plasma polymers, which accords with empirical observations in many studies that plasma coatings with higher functionality could be obtained at low power inputs. Our data show that this is not so much a function of changes in the plasma bulk phase; the mass spectra do not differ markedly. Rather, the main effect arising from the use of low power is that the acceleration of ions traversing from the bulk plasma through the sheath onto the surface is significantly reduced. As shown by the ion energy distributions, from $10 \mathrm{~W}$ plasmas the protonated molecular ions arrive at the surface with much lower energies than from $50 \mathrm{~W}$ plasmas and hence are able to soft-land. This leads to a substantial reduction of the likelihood of the ions fragmenting upon impact and hence to improved retention of functionality.

The significance of our results extends, however, well beyond confirming the utility of low power inputs. Clearly, the use of low power is by itself not sufficient for creating optimally functionalized plasma polymers. A high density of monomer molecular ions in the plasma phase is another key parameter; by protonating the monomer molecule the structure appears to be stabilized and protected, to some extent, from fragmentation by collisions. If the monomer molecule can be protonated efficiently and transported to the surface of the substrate/growing 
film, and does not fragment upon impact due to a high IED, we obtain a promising scenario for transporting intact functionality from the plasma phase into the growing plasma polymer film.

This is where pressure comes in as a key variable. It is clear from the above data that the efficiency of generation of the protonated monomer ion, the IED, and the ion flux (of the protonated monomer ion) to the surface all vary with pressure. Our data show that optimal conditions exist in the pressure region where the plasma transitions between the $\alpha$ and $\gamma$ phases. At low pressure, in the $\alpha$ plasma phase, plasma-phase mass spectra show a higher extent of fragmentation of the monomer molecule, with a diversity of fragment ions. While protonated monomer ions do exist in the plasma bulk phase, their acceleration through the sheet confers substantial kinetic energy, leading to substantial probability of fragmentation on impact on the growing plasma polymer surface. At pressure values corresponding to the $\gamma$ plasma phase, on the other hand, there is high relative abundance of the protonated monomer ion in the plasma phase and acceleration through the sheath confers less energy due to the occurrence of collisions. Collisions while traversing the sheath are thus beneficial in reducing the IED, but they also reduce the ion flux arriving at the surface, thus reducing the relative contribution of ions to the plasma polymer growth compared with radical processes.

It is therefore under pressure conditions in the transitional regime that an optimal compromise exists: a relatively high abundance of protonated monomer ions, sufficient collisions to avoid an excessively high IED, yet not so many collisions that the ion flux becomes stifled.

It also can be inferred that plasma bulk processes, and their control via power and pressure, do play a role, but more critical is understanding and control of the transport of ions through the plasma sheath.

\subsection{Ion flux}

It is clear from the above discussion that control of the relative abundance and the IED of ions can be used to enhance the probability of incorporating desired chemical structures into plasma polymers, but one question remains: is the flux of protonated monomer ions to the surface sufficient to lead to a significant role of these ions, and their intact chemical groups, in the formation of plasma polymers? If the plasma polymer was formed predominantly via radical processes, then optimising the abundance in the plasma phase of desired ions and their IED might make little difference. A favourable aspect, though, is that the acceleration of ions through the sheath, versus diffusion-controlled transport of radical species, enhances the ionic contribution relative to their abundance in the plasma bulk phase. Our XPS and ToF-SIMS data 
correlating with the abundance of the protonated monomer ion in the plasma phase (Figures 2 and 7) suggests that indeed these ions play a major role in the film formation. To gain a better appreciation, we have measured the ion flux to the collector surface.

The total ion mass flux to the collecting surface was estimated using the ion flux (Table 1) and the average ion mass from the positive mass spectra, analogous to the method used in an earlier study [19]. The ion mass flux was then compared to the total mass flux measured using a quartz crystal microbalance. Table 1 shows that the mass flux of ions substantially exceeds the deposition rate of the plasma polymers under all pressure conditions studied. This finding shows that ions can indeed contribute a substantial percentage of the accumulated film mass, as found in earlier studies [28,29]. The data also show that the sticking probability of the arriving ions is $<<1$, and fragmentation might also lead to parts of impacting ions being lost to film growth. In particular, stable fragments such as $\mathrm{CO}, \mathrm{CO}_{2}$ and $\mathrm{H}_{2} \mathrm{O}$ could be eliminated upon collision with the surface; the reduction in carbonyl content evident from XPS data indicates that some of the ester groups lose $\mathrm{CO}$ and perhaps $\mathrm{CO}_{2}$ in the plasma and/or on impact on the surface. The sticking probability of ions depends on the ion energy and chemistry, but is often in the range of $20-50 \%$ [24]. Our data suggest a significantly lower sticking probability, although at present quantitative estimation seems premature. Pending further work we can conclude that there is a considerable flux of ions (as well as of radicals) and it appears reasonable to postulate that they play a major role in film formation and incorporation of functional groups. When the protonated monomer ion is a major component of the ions produced in the plasma (Fig. 1), plus soft-landing and a high ion flux, ideal conditions exist for incorporating substantial amounts of desired structural elements such as ester groups.

Table 1: Ion flux, ion mass flux and deposition rates for ETMA plasma polymerization at various pressures.

\begin{tabular}{|l|l|l|l|}
\hline $\begin{array}{l}\text { Pressure } \\
(\mathrm{mbar})\end{array}$ & $\begin{array}{l}\text { Ion flux } \\
\left(10^{18} \mathrm{ions} / \mathrm{m}^{2} \mathrm{~s}\right)\end{array}$ & $\begin{array}{l}\text { Ion mass flux } \\
\left(\mu \mathrm{g} / \mathrm{m}^{2} \mathrm{~s}\right)\end{array}$ & $\begin{array}{l}\text { Deposition rate } \\
\left(\mu \mathrm{g} / \mathrm{m}^{2} \mathrm{~s}\right)\end{array}$ \\
\hline $1.1 \times 10^{-1}$ & 2.3 & 445 & 30 \\
\hline $4 \times 10^{-2}$ & 3.4 & 754 & 47 \\
\hline $1 \times 10^{-2}$ & 2.6 & 683 & 95 \\
\hline
\end{tabular}




\subsection{Identification of Plasma Regimes}

In this section we will discuss ways of estimating the $\alpha$ to $\gamma$ transition and thus to home in on approximate optimal plasma conditions for functional group retention via ions, when the above approach involving plasma mass spectrometry, correlation with XPS and ToF-SIMS, and ion energy distribution measurements is not available. This may also be of value in reducing the issue of results of plasma polymerization varying between laboratories with different designs of plasma reactors [30,31]. To predict outcomes for reactors of different designs the intrinsic plasma processes occurring in the plasma phase, in the sheath, and at the substrate surface must be measured and therefore plasma diagnostics are required. But qualitative estimation is feasible using simpler considerations.

Plasmas can be sustained in two distinct regimes. In the $\alpha$ regime the density of particles is low and the mean free path is long, so the plasma is sustained by the acceleration of bulk electrons. Increasing the pressure to the $\gamma$ regime yields a collisional sheath. liberating electrons in the sheath where they will be heated by the strong electric field. The plasma density near surfaces increases with respect to the bulk plasma higher ion flux to the surface results. However, the ion energy is reduced due to ion-neutral collisions on approach to the surface. Hence, operating the plasma on either side of the $\alpha \rightarrow \gamma$ transition may result in very different processes at the surface and therefore plasma polymer deposits with different properties.

Identification of plasma regimes and estimation of the transition point can be done using a number of different approaches: spatial distribution of the plasma, gas pressure, Ohmic RF current, phase angle [32], and ion energy distribution. These approaches are illustrated in Figure 7 with example data recorded with ETMA plasmas, except for ion energy distribution, which has been discussed above.

\subsubsection{Spatial distribution of Plasma Glow}

With increasing pressure, the plasma glow zone contracts. Figure 8 shows plasma glows in $\alpha$ and $\gamma$ regimes. The glow close to sheath boundaries is a bright light grey colour in the $\gamma$ mode, indicating the existence of high energy electrons accelerated by a high voltage drop across the sheath. On the other hand, a uniform glow is observed of the quasi-neutral plasma in the $\alpha$ mode. Concurrent changes to the discharge appearance close to the sheath boundaries, accompanied by changes to the current-voltage characteristics, is an indication of the $\alpha \rightarrow \gamma$ plasma transition. 

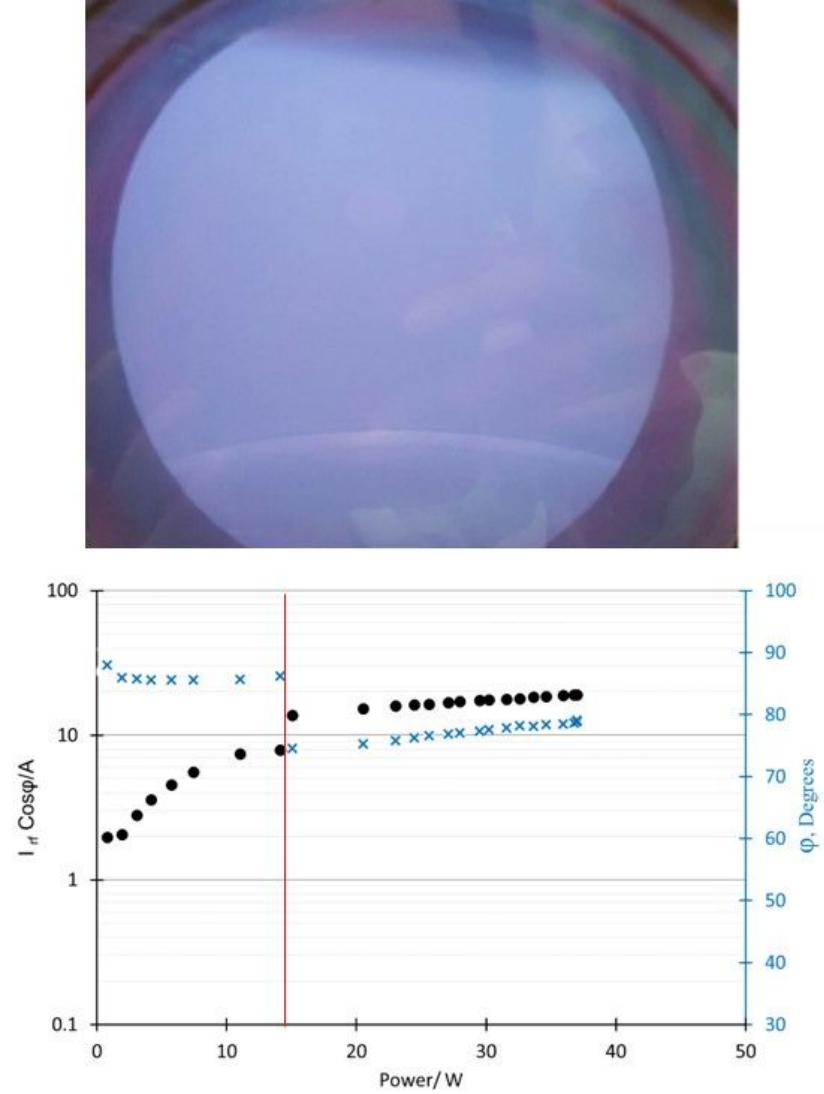
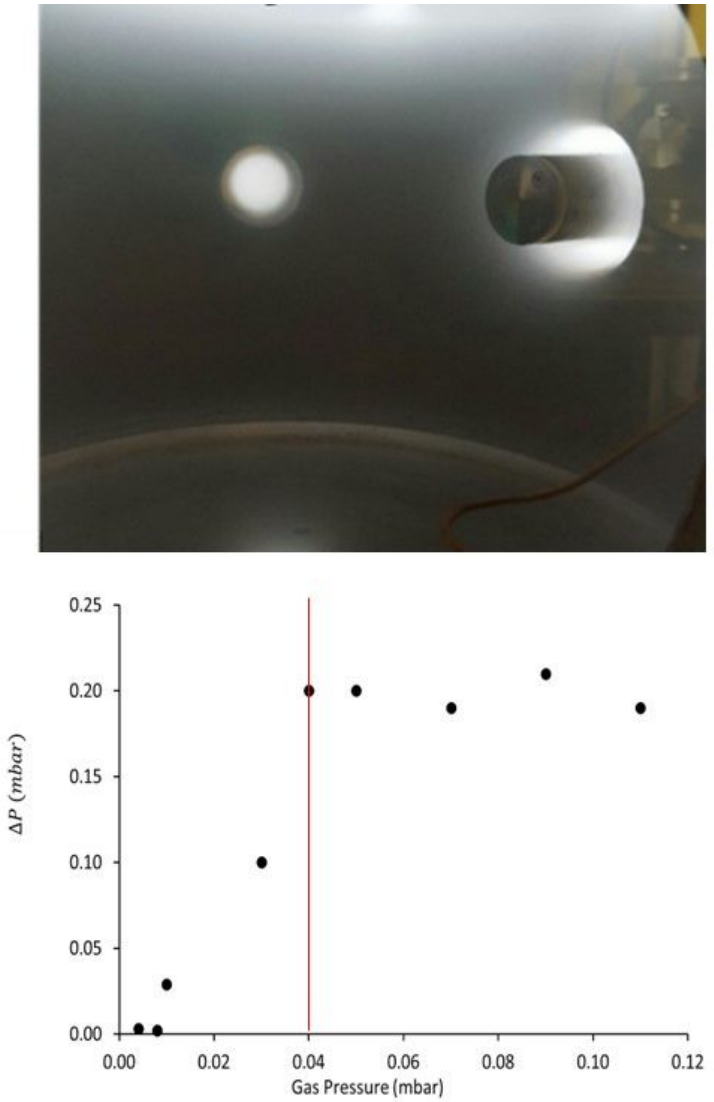

Figure 8. Approaches to identification of plasma regimes: (top) visual observation of the plasma glow in $\alpha$ (left) and $\gamma$ (right) regimes; (bottom left) phase angle and Ohmic rf current (Irf $\cos \varphi$ ) as a function of applied rf power. The phase angle is of the measured current vector with respect to the measured voltage vector and is expressed in degrees. (bottom right) change in pressure as a function of initial gas pressure, for 50W ETMA plasma. Red lines indicate the transitions between $\alpha$ and $\gamma$ regimes

\subsubsection{Change in pressure upon ignition}

Usually, upon ignition the pressure inside a plasma chamber changes as dissociation and polymerization reactions occur. The extent of change also depends on factors such as chamber configuration, but the case of ETMA demonstrates that there is a much more pronounced change in pressure when the plasma is operated in the $\gamma$ mode than in the $\alpha$ mode (Figure 7). Thus, plotting the pressure change as a function of initial (pre-ignition) gas pressure can be used to establish the transition zone between these plasma regimes.

\subsubsection{Ohmic RF current}

Figure 7 also shows the phase angle $(\varphi)$ and the Ohmic current as a function of applied power for ETMA plasma. As for the change in pressure, there is a discontinuity in the behaviour of 
these observables and again this can be used to approximate optimal conditions suitable for functional group retention. At low RF power the plasma density and conductivity are small, therefore the resistance is comparable to the capacitive impedance of the sheaths. Furthermore, the contribution of the ohmic current to the total discharge current is at maximum, and the phase angle approaches a minimum value. When a discharge does not exist, the RF current is only limited by the capacitive reactance of the gap between the electrodes. In the presence of a discharge with a low plasma density, the resistance of the plasma is significant, whereas for a high plasma density and as a consequent high conductivity, the RF current is restricted by the sheaths' capacitive reactance, which depends on the sheath's thickness. In other words, with increasing plasma density, the sheath thickness and its ohmic resistance of the quasineutral plasma decrease considerably, whereas the capacitive reactance changes little.[33]

\subsubsection{Ion Energy Distribution Function}

The IED function is perhaps the most useful parameter as it can be used to monitor the alpha to gamma transition and thus can be used to assess the nature of the plasma. The ion energy distributions of the precursor ions are shown in Figures 1 and 3. At low pressure the ion energy distributions are narrow, indicative of a collisionless sheath, whereas broad distributions were observed when the pressure was increased. In a collisional sheath, ions gain energy from the electrical potential drop but lose energy before they arrive at the surface due to neutral molecules collisions. Consequently a broad distribution of ion energies is seen in the collisional regime.

\section{CONCLUSIONS}

Using the monomer ethyl trimethylacetate, plasma polymers were deposited under a range of conditions with concurrent analysis of the plasma gas phase. Mass spectrometry of the plasma phase showed the presence of protonated molecular ions, and surface analysis of coatings by XPS and ToF-SIMS showed that intact ester groups were incorporated into the polymer films, whilst also showing that the ETMA film compositions were determined by activation in the plasma phase of both radicals and heavy ions. Varying pressure and RF power caused marked variations in the composition of ions in the plasma phase and the contribution of intact molecular ions to deposition and functionality. Converting a plasma from collisionless to collisional sheaths markedly alters the chemistry of the plasma and the resultant plasma polymer. 
However, the relative abundance of molecular ions is not predictive of the extent of incorporation of the desired ester functionality into the resultant plasma polymers. The ion flux and average ion energy are critical factors to achieve retention of ester groups through the plasma process; while achieving the required high flux of intact molecular ions, these ions should approach the surface of the substrate and the growing coating with an average energy suitable for soft landing. The compromise between abundance of protonated molecular ions and their ion energy distribution is optimal near the transition between the $\alpha$ and $\gamma$ plasma phases. RF power input also affects the relative abundance of the protonated molecular ion and fragment ions, but the main effect arising from the use of low power is that the acceleration of ions traversing from the bulk plasma through the sheath onto the surface is much reduced and hence ions are able to soft-land.

Thus, optimal conditions for functional group incorporation into plasma polymers were determined to include a high abundance of protonated molecular ions in the plasma phase combined with a high ion flux and soft-landing conditions. By measuring the ion energy distribution it is feasible to identify optimal plasma polymerization conditions. Alternatively, when characterization of ion energy distribution is not available, simpler analytical methods can be used for guided identification of the optimal plasma regime. Predicting the parameters at which the transition occurs for each precursor is not trivial due to the variety of processes which occur and the collisional cross-sections of the various species, but analytical methods can be used for guided identification of the optimal plasma regime and enable rational selection of plasma conditions near the transition between $\alpha$ and $\gamma$ phases, where we can expect optimal retention of the desired functional group.

\section{ACKNOWLEDGMENT}

This work was funded in part by the Australian Government under ARC Discovery project DP 160105001.

\section{Data Availability Statement}

The data that support the findings of this study are available from the corresponding author upon reasonable request. 


\section{REFERENCES}

1. B. Thierry, M. Jasieniak, L.C. de Smet, K. Vasilev and H.J. Griesser, Reactive epoxyfunctionalized thin films by a pulsed plasma polymerization process. Langmuir, 2008. 24, 10187.

2. L. O'Toole, A.J. Beck and R.D. Short, Characterization of plasma polymers of acrylic acid and propanoic acid. Macromolecules, 1996. 29, 5172.

3. C.A. Mayhew, and R.D. Short, Selected ion flow tube studies to investigate the formation of acrylic and propionic acid protonated clusters in low power, low pressure RF plasmas. Chem. Commun. 2009, 6, 659.

4. X. Zou, E. Kang, K. Neoh, Y. Zhang, K. Tan, C. Cui, and T. Lim, Plasma polymerization and deposition of glycidyl methacrylate on $\mathrm{Si}$ (100) surface for adhesion improvement with polyimide. Polymers for Advanced Technologies, 2001. 12, 583.

5. L.M. Szott, M.J. Stein, B.D. Ratner and T.A. Horbett, Complement activation on poly (ethylene oxide)-like radiofrequency glow discharge-deposited surfaces. Journal of Biomedical Materials Research Part A, 2011. 96, 150.

6. V. Panchalingam, X. Chen, H. Huo, C. Savage, R. Timmons, and R. Eberhart, Pulsed plasma discharge polymer coatings. ASAIO journal, 1993, 39, M305.

7. D. Beyer, W. Knoll, H. Ringsdorf, J.H. Wang, R.B. Timmons, and P. Sluka, Reduced protein adsorption on plastics via direct plasma deposition of triethylene glycol monoallyl ether. Journal of Biomedical Materials Research Part A, 1997, 36, 181.

8. G. Padron-Wells, B.C. Jarvis, A. Jindal and M. Goeckner, Understanding the synthesis of DEGVE pulsed plasmas for application to ultra thin biocompatible interfaces. Colloids and Surfaces B: Biointerfaces, 2009, 68, 163

9. M.R. Alexander, and T.M. Duc, A study of the interaction of acrylic acid/1, 7-octadiene plasma deposits with water and other solvents. Polymer, 1999, 40, 5479.

10. E. Makhneva, A. Manakhov, P. Skládal and L. Zajíčková, Development of effective QCM biosensors by cyclopropylamine plasma polymerization and antibody immobilization using cross-linking reactions. Surface and Coatings Technology, 2016, 290, 116 
11. D. Hegemann, B. Hanselmann, S. Guimond, G. Fortunato, M.-N. Giraud, and A.G. Guex, Considering the degradation effects of amino-functional plasma polymer coatings for biomedical application. Surface and Coatings Technology, 2014, 255, 90

12. H. Yasuda, Plasma polymerization. 1985: Academic press.

13. A. Choukourov, J. Kousal, D. Slavínská, H. Biederman, E.R. Fuoco, S. Tepavcevic, J. Saucedo, and L. Hanley, Growth of primary and secondary amine films from polyatomic ion deposition. Vacuum, 2004, 75, 195

14. L. Hanley, E. Fuoco, M.B. Wijesundara, A.J. Beck, P.N. Brookes, and R.D. Short, Chemistry and aging of organosiloxane and fluorocarbon films grown from hyperthermal polyatomic ions. Journal of Vacuum Science \& Technology A, 2001. 19, 1531

15. R.V.F. Paulino, S. Saboohi and A. Michelmore, The chemistry of organophosphate thin film coatings from low pressure plasma and the effect of the substrate on adhesion. Plasma Processes and Polym. 2017, 14, e1700037

16. S. Saboohi, M. Jasieniak, B.R. Coad, H.J. Griesser, R.D. Short, and A. Michelmore, Comparison of Plasma Polymerization under Collisional and Collision-less Pressure Regimes. J. Phys. Chem. B, 2015, 119, 15359

17. S. Saboohi, B.R. Coad, H.J. Griesser, A. Michelmore and R.D. Short, Synthesis of highly functionalised plasma polymer films from protonated precursor ions via the plasma $\alpha-$ $\gamma$ transition. Phys. Chem. Chem. Phys., 2017, 19, 5637

18. S. Saboohi, B.R. Coad, A. Michelmore, R.D. Short and H.J. Griesser, Hyperthermal Intact Molecular Ions Play Key Role in Retention of ATRP Surface Initiation Capability of Plasma Polymer Films from Ethyl $\alpha$-Bromoisobutyrate. ACS Applied Materials \& Interfaces, 2016, 8,16493

19. D. Barton, A.G. Shard, R.D. Short and J.W. Bradley, The effect of positive ion energy on plasma polymerization: a comparison between acrylic and propionic acids. J. Phys. Chem. $B, \mathbf{2 0 0 5}, 109,3207$

20. A. Michelmore, D.A. Steele, J.D. Whittle, J.W. Bradley and R.D. Short, Nanoscale deposition of chemically functionalised films via plasma polymerisation. RSC Advances, 2013. 3,13540 
21. D. Bohm, In The Characteristics of Electrical Discharges in Magnetic Fields; Guthrie, A., Wakerling, R. K., Eds.; McGraw Hill: New York, 1949.

22. M.A. Lieberman, A. J. Lichtenberg, Principles of Plasma Discharges and Materials Processing, 2nd Ed.; John Wiley and Sons: Hoboken, NJ, 2005.

23. S. Saboohi, R.D. Short, B.R. Coad, H.J. Griesser and A. Michelmore, The Physics of Plasma Ion Chemistry: A Case Study of Plasma Polymerization of Ethyl Acetate, J. Phys. Chem. Lett., 2019, 10, 7306

24. S. Saboohi, H.J. Griesser, B.R. Coad, R.D. Short and A. Michelmore, Promiscuous Hydrogen in Polymerising Plasmas, Phys. Chem. Chem. Phys., 2018, 20, 7033

25. D.C. Jacobs, Reactive collisions of hyperthermal energy molecular ions with solid surfaces. Annu. Rev. Phys. Chem., 2002, 53, 379

26. M.B. Wijesundara, Y. Ji, B. Ni, S.B. Sinnott and L. Hanley, Effect of polyatomic ion structure on thin-film growth: Experiments and molecular dynamics simulations. J. Appl. Phys., 2000, 88, 5004

27. P.N. Brookes, S. Fraser, R.D. Short, L. Hanley, E. Fuoco, A. Roberts, and S. Hutton, The effect of ion energy on the chemistry of air-aged polymer films grown from the hyperthermal polyatomic ion Si 2 OMe 5+. Journal of Electron Spectroscopy and Related Phenomena, 2001, 121, 281

28. C. Mayhew, and R. Short, On the plasma polymerisation of allyl alcohol: an investigation of ion-molecule reactions using a selected ion flow tube. J. Chem. Soc., Faraday Trans., 1997, 93, 1961

29. L. O'Toole, R.D. Short, A.P. Ameen and F.R. Jones, Mass spectrometry of and deposition-rate measurements from radiofrequency-induced plasmas of methyl isobutyrate, methyl methacrylate and n-butyl methacrylate. J. Chem. Soc., Faraday Trans., 1995, 91, 13631370.

30. A. Michelmore, C. Charles, R.W. Boswell, R.D. Short and J.D. Whittle, Defining plasma polymerization: new insight into what we should be measuring. ACS applied materials \& interfaces, 2013, 5, 5387

31. J.D. Whittle, R.D. Short, D.A. Steele, J.W. Bradley, P.M. Bryant, F. Jan, H. Biederman, A.A. Serov, A. Choukurov, A.L. Hook, W.A. Ciridon, G. Ceccone, D. Hegemann, E. Korner, 
A. Michelmore, Variability in Plasma Polymerization Processes-An International Round-Robin Study. Plasma Processes and Polymers, 2013, 10, 767

32. C. Rawlins, Basic AC circuits. 2000: Elsevier.

33. V. Lisovskiy, J.-P. Booth, K. Landry, D. Douai, V. Cassagne, and V. Yegorenkov, Modes and the alpha-gamma transition in $\mathrm{rf}$ capacitive discharges in $\mathrm{N}_{2} \mathrm{O}$ at different $\mathrm{rf}$ frequencies. Physics of Plasmas, 2006, 13, 103505 
Graphical Abstract:
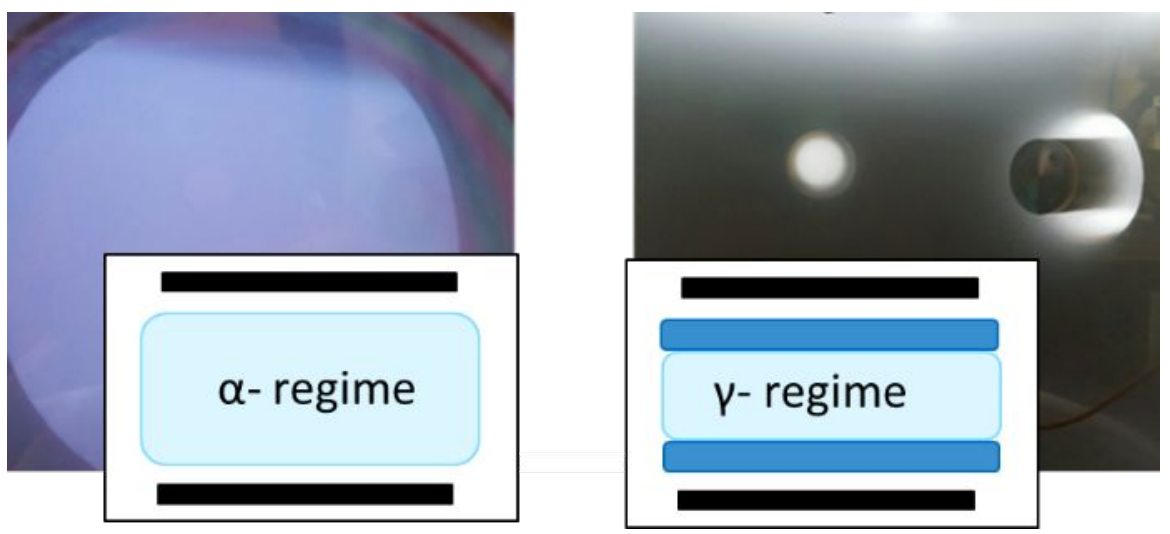
Manuscript ppap.202000195 Saboohi et al - Graphical abstract
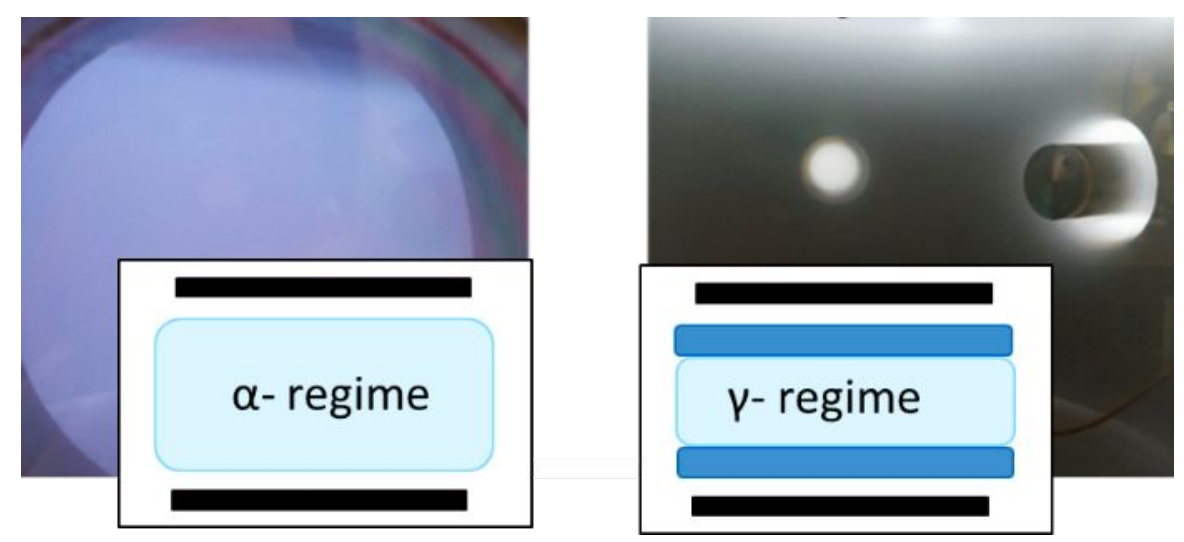